Fission of Uranium under Deuteron Bombardment

SrNCE the phenomenon of neutron-induced fission of the uranium and thorium nuclei is now indisputably established, attention may be turned to the possible efficacy of other bombarding particles in this respect. An outline is here presented of evidence suggesting that high-energy deuterons are, in fact, capable of producing fission in uranium.

The following experimental arrangement has been used. One inside surface of a copper box is covered with a $20 \mathrm{mgm} . / \mathrm{cm} .^{2}$ layer of uranium metal, and is bombarded by deuterons passing through an aperture in the opposite side of the box. An inside pocket on one of the other walls contains metal foils for the purpose of collecting particles projected from the target through an aperture in the pocket. A thickness of $2 \mathrm{~mm}$. S.P. aluminium is always present to protect the collecting foil from the low-energy products due to mechanical disintegration of the target. Bombard. ments of about l $\mu \mathrm{amp}$. for ten minutes are convenient, and are found to produce on the collector activities of intensity suitable for measuring with a Geiger counter.

It is necessary to estimate what part of the activity on the collector is due to general neutron radiation and to deuterons scattered from the target. In the pocket are placed three foils of $3.3 \mathrm{mgm} . / \mathrm{cm}^{2}$ aluminium, $F, D$ and $N$ in order of proximity to the target, and a single thickness of half-millimetre copper sheet between the foils $D$ and $N$. Five minutes after a 4 ramp./min. bombardment with $9 \mathrm{Mv}$. deuterons, it is found that the neutron effect of the irradiation has produced in $N$ a small activity of 40 counts per minute; the neutron effect is eliminated from $D$ and $F$ simply by subtraction of this $N$ effect. $D$ 's activity measures the scattered-deuteron effect and is initially about 100 counts per minute, decay. ing with a half-life of $2 \cdot 3 \mathrm{~min}$. to reach a negligibly small value after $20 \mathrm{~min}$. F's activity, however, is about 1,500 counts per min., and is much longerlived, although with a target entirely of lead, $F$ as well as $D$ shows only a $D$ effect.

The decay curve for F's activity has been followed for about 7 hours and has been compared with that calculated from Frisch's formula ${ }^{1}$ for the decay of multiplex activity. The measure of agreement is so substantial as to preclude the possibility of a merely accidental coincidenco.

A stack of six $0.66 \mathrm{mgm} . / \mathrm{cm} .{ }^{2}$ aluminium foils has been placed in tho pocket and after the lapse of 30 minutes (when the $D$ effect has decayed) the activities are found to decrease regularly through the stack, reaching inappreciably small values in the fifth and sixth foils. Regarding another aspect, the fact that replacement of aluminium as the collector material by silver causes no significant difference in the decay curvo, suggests that the activity has been implanted rather than induced. The evidence thus indicates that radioactive nuclei are projected from the uranium target with a range of the order of $2 \mathrm{~cm}$.; this occurrence is evidently to be ascribed to a fission of the uranium nucleus.

It is important to assess the magnitude of the fission effect caused by background neutron radiation, and for this purpose there has been enclosed in the pocket itself a layer of uranium, separated from $F$ by $2 \mathrm{~mm}$. S.P. of aluminium, and from the target by the copper sheet, this arrangement necessarily reproducing fairly closely that activity in $F$ which is due to neutron-induced fission. The intensity in this case is less than one twentieth of that observed in the positive experiment.
Approximate measurements have been made on the excitation function for deuteron-induced fission, using aluminium absorbers to modify the energy of the beam and a suitable disposition of diaphragms to protect the collector against deuterons scattered from the absorbors. The threshold for the process appears to lie at about $8 \mathrm{MV}$., and the cross-section increases rapidly in the range 8-9 Mv.

The conclusions drawn from these preliminary experiments would receive unequivocal confirmation from a chemical identification of the fragment nuclei or a detection of their large ionization impulses in an ionization chamber. It might also prove profitable to examine the effects given by others of the heaviest elements (especially thorium).

Dr. R. S. Krishnan and other colleagues of the Cavendish cyclotron group have rendered me indispensable assistance, and I am indebted also to Prof. $J$. D. Cockeroft for pertinent suggestions.

Cavendish Laboratory.

Cambridge. Aug. 26.

S NATURE, 148, 852 (1939).

\section{Absorption of Polymolecular Films in the Infra-Red}

THE use of infra-red absorption spectra as a means of investigating molecular structure has still to be extended to surface films. Until very recently, the possibilities of this extension seemed remoto, since a monomolecular layer does not contain enough molecules per square centimetre to produce moasurable absorption. Thus it is found experimentally that approximately $10^{19}$ molecules are required to produce appreciable absorption in a beam of $1 \mathrm{sq} . \mathrm{cm}$. cross-section, whereas tho number of molecules per square centimetro in a monomolecular layer is about $5 \times 10^{14}$. With the production of polymolecular films close to 1,000 molecules thick, tho possibility of. detecting absorption still seemed slight, but technically feasiblo, if a method employing several reflections through such a film were employed.

As a matter of interest, it was decided to try whether any absorption could bo detected using a single reflection through a film of methyl stearate 700 molecules thick, deposited on a chromium-plated strip of metal. Wo were surprised to find that the well-known absorption at $3 \cdot 3 \mu$ due to $\mathrm{CH}$ groups was easily detectablo, showing about 30 per cent absorption, while other weaker bands were noticeable between $6 \mu$ and $10 \mu$. Next, a film only 200 mole. cules thick was tried. This still gave the $3 \cdot 3 \mu$ band with appreciable intensity. These results mean that absorption spectra were being obtained from ap. proximately $10^{17}$ molecules, instead of the customary 1019. In other words, the absorption coefficient of a moleculo in the infra-red seems to be considerably increased when it is in a surface film.

The possible causes of this will not be discussed here. The purpose of this note is merely to record this fact, which opens up a new and potentially interesting field of research in infra-red spectroscopy and its application to problems of molecular structure. In particular, this may prove to be a very suitable method of studying protein molecules.

Wo wish to express our thanks to Dr. Stenhagen, who prepared the films.

G. B. B. MI. SutherLand.

IV. T. TUTTE.

Laboratory of Physical Chemistry, Cambridge. Sept. 1. 